\title{
Vibration monitoring of long bridges and their expansion joints and seismic devices
}

\author{
Kleidi Islami ${ }^{1}$ \\ ${ }^{1}$ mageba SA, Department of SHM, Solistrasse 68, 8180 Bülach, Switzerland
}

\begin{abstract}
This paper presents a number of recently installed Structural Health Monitoring (SHM) systems: a) on a $2 \mathrm{~km}$ double suspension bridge; b) on a long railway viaduct that has experienced cracking; and c) on a steel arch bridge in a seismically active area. Damage detection techniques have been applied based on high-frequency measurements of vibrations, pressure and strain, enabling a proper understanding of the structures' behaviour to be gained. The diverse range of applications presented, designed in collaboration with structure owners and design engineers, includes damage detection on expansion joints of suspension bridges, crack analysis and correlation with accelerations of high-speed trains, and high-frequency performance monitoring of seismic devices. These case studies, based on both static and dynamic approaches, demonstrate the usefulness and ease of use of such systems, and the enormous gains in efficiency they offer.
\end{abstract}

\section{Introduction}

Monitoring of structures by advanced technologies that allow real-time observations and permit to respond effectively in case of critical conditions is nowadays established practice. Such practice generally takes the form of automated Structural Health Monitoring systems. SHM systems typically consist primarily of a data acquisition unit and a number of sensors, together with data processing and data transmission capabilities as may be required.

The implications of the development of techniques of monitoring, of statistical modelling of the response of structures, and of gathering and processing data in real time, are important - especially in the context of particularly sensitive structures. It is desirable to verify whether the effects of various environmental variables measured in situ influence the static or dynamic behaviour of the structure. Therefore, it is important to eliminate the influence of these factors, so that small changes due to damage can be detected. This is made possible, for example, by the use of regression models, which can determine the static variables starting from a predefined input.

These techniques shall be described below, with particular reference to case studies from a suspension bridge, a long concrete viaduct and a retrofitted arch bridge.

For example, $25 \mathrm{kHz}$ vibrations are measured at the expansion joints of a double suspension bridge, enabling damage or deterioration to be detected at an early stage by recording the level of accelerations and natural frequencies under traffic. The availability of such systems has now led to the development of "smart" expansion joints: expansion joints that feature an integrated advanced monitoring system, already when fabricated.

The second case study illustrates the monitoring of a recently built high-speed railway viaduct subjected to early cracking. Extensive analyses have been performed, and correlations between crack openings and accelerations under high-speed trains, in particular, have shown that the cracking has largely discontinued.

Finally, the seismic retrofitting of an arch bridge has been tested by an SHM system permanently measuring accelerations of the deck and pressure inside the shock transmission units (STU) at $100 \mathrm{~Hz}$ frequency. Analysis of the data shows, that the seismic devices are sensitive also to vehicles crossing the bridge This was shown by pressure signals coming from the eight STUs rigidly connected to the structure and the accelerometers.

These non-standard monitoring applications for civil structures demonstrate the usefulness of SHM in assessing and maintaining not only an entire structure but also its key functional elements.

\section{Monitoring of the Taizhou Bridge, Jiangsu province, China}

The Taizhou Yangtze River Bridge (Figure 1), newly constructed at a cost of USD 400 million, is the world's longest-span bridge of its type. The three-tower suspension bridge, with two main spans of $1,080 \mathrm{~m}$ each and side spans of $390 \mathrm{~m}$, crosses the Yangtze River where it has a width of $2.1 \mathrm{~km}$ (Chen \& Jianchi 2012). This extraordinary bridge required some extraordinary

\footnotetext{
${ }^{1}$ Corresponding author: kislami@mageba.ch
} 
key components, such as the expansion joints which accommodate deck movements while providing a driving surface for traffic. Modular expansion joints with 18 gaps each (and thus capable of accommodating 1,440 $\mathrm{mm}$ of longitudinal movement) were installed at each end of the deck (Figure 1).
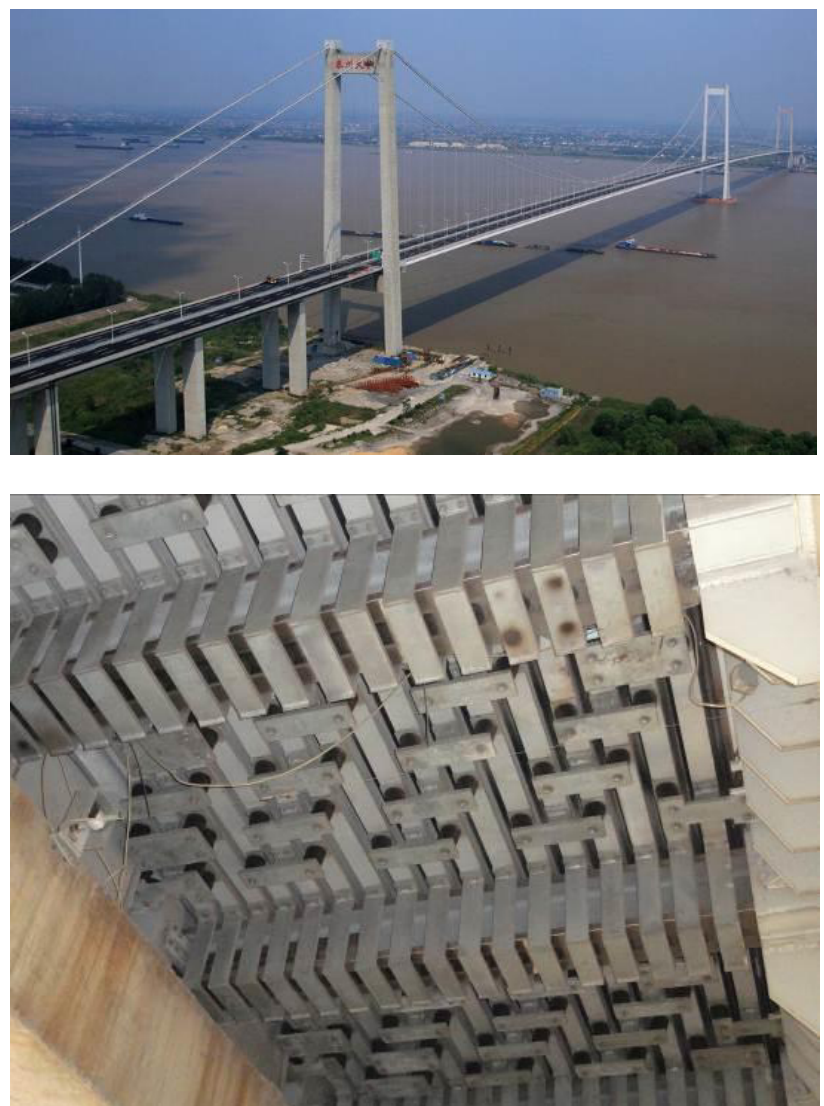

Figure 1. The new Taizhou Yangtze River Bridge (top) and one of its modular expansion joints, viewed from below (bottom).

An SHM system was installed on the bridge (Islami et al., 2014) to provide the type of data on the bridge's condition that is likely to be a great interest to any owner whose structure is exceptional in some way. The basic system measures and records the movements and rotations of the deck at the expansion joints, and thus gives a valuable impression of the performance of the structure at any time, enabling the need for maintenance or adaptation work to be quickly identified and planned. It also reports accumulated sliding movements over time - a key indicator in evaluating and predicting the condition of key mechanical components such as expansion joints and bearings.

An example of the recorded data is presented in Figure 2, showing overall displacements and the correlation between displacements of a particular lamella beam (on the surface of an expansion joint) and the overall movements of the bridge. A $45^{\circ}$ inclination of the correlation graph would indicate that these values are equal.
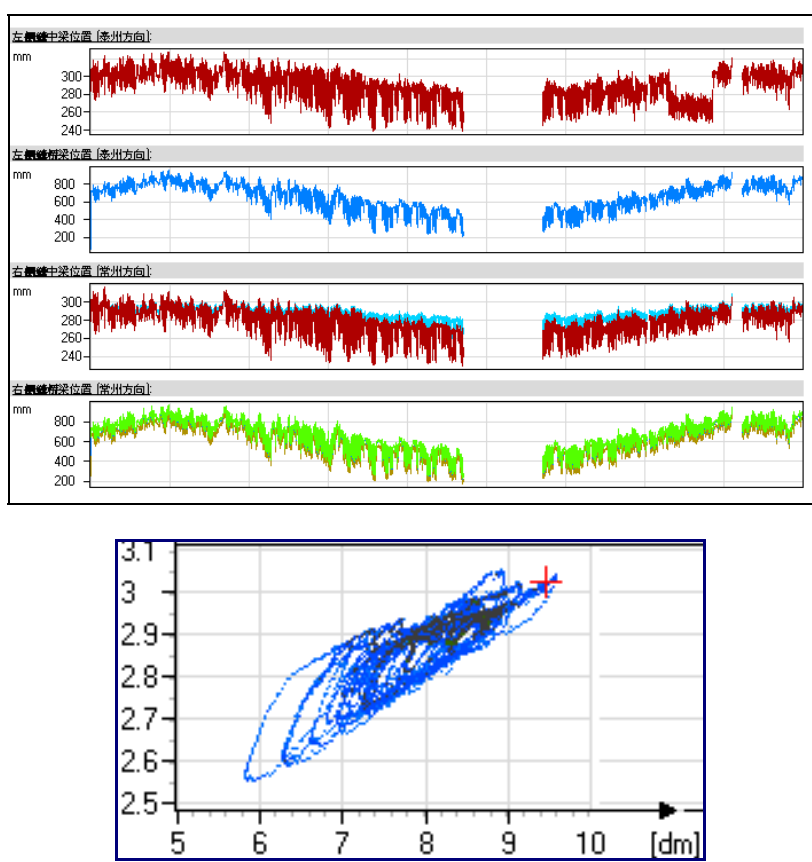

Figure 2. Overall movements (top) and correlation between lamella beam and bridge movements (bottom).

To maximise in particular the system's ability to monitor the condition and performance of the expansion joints perhaps the parts of the bridge which deserve the most inspection and maintenance attention - an upgrade to the monitoring system has been applied. A brand new damage detection feature has been installed, incorporating sensors at the joints which will provide clear information about the condition of the joints and support the planning of maintenance activities. The functioning of the new feature is based on the measurement of structure-borne vibrations recorded at a sampling frequency of $25.6 \mathrm{kHz}$, with even very tiny changes in the joint or its performance being detectable and visually represented by changes in a curve on a graph.

The installed "smart" expansion joint (Figure 3) measures high-frequency movements, inclinations, temperature and vibrations enabling a proper understanding of the joint's and bridge's behavior to be gained. The main purpose of the project is not only to monitor the condition and performance of the expansion joints due to extensive movement or rotation (basic function), but also to detect damage at an early stage by recording the level of accelerations and natural frequencies caused by traffic (advanced function).

As a first step, many artificial failures (Figure 3) were created in order to simulate damage. The different setups of sensors mostly covered all of the joist beams that support the lamella beams of the expansion joint. During the tests there was a good distinction, by the system, between damaged and undamaged joint conditions. This enabled the system to be fine-tuned before permanent installation. The permanent monitoring sends data to a remote server, including daily levels of vibrations due to heavy traffic together with the associated modal frequencies. 


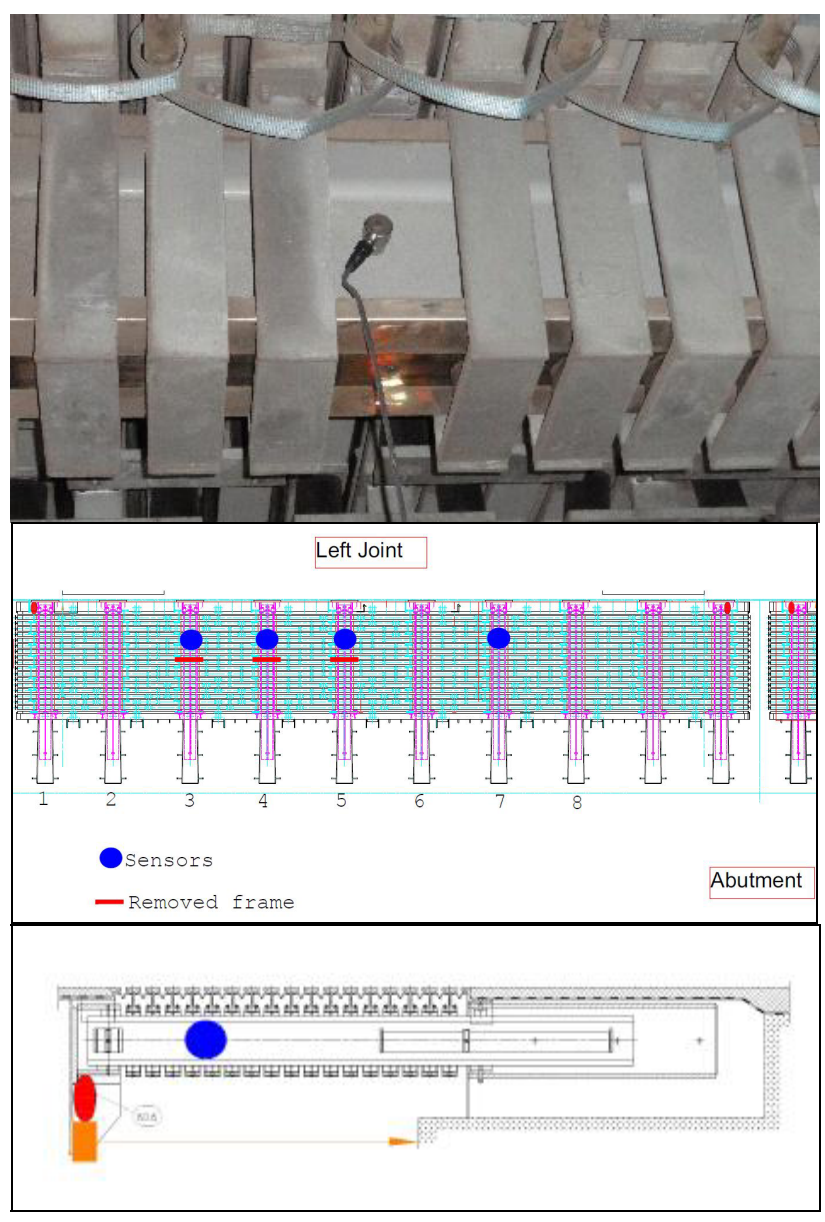

Figure 3. Preliminary vibrational tests and modal analysis on the large expansion joint. Layout of sensors and fault simulation.

The automatic procedure implemented in the system determines the thresholds based on statistical analyses that define the vibration limits by avoiding false alarms. Each type of damage causes a notable repeated hammering on the structural parts of the expansion joints, enabling the sensors installed on the joist beams to detect it immediately. Once this limit is exceeded, it indicates the occurrence of damage, causing an alarm notification to be sent by email and to appear on the system's web interface. The next step is a site visit in order to verify the damage and avoid further damage or deterioration.

This new application will provide clear information about the condition of the joints and support the planning of maintenance activities. Different types of damage can be clearly identified based on general testing and teaching of the system. This will allow very sensitive, but also reliable, damage identification. As a result, unexpected damage can be immediately recognised and notified, enabling the timing of replacement of components to be optimised.
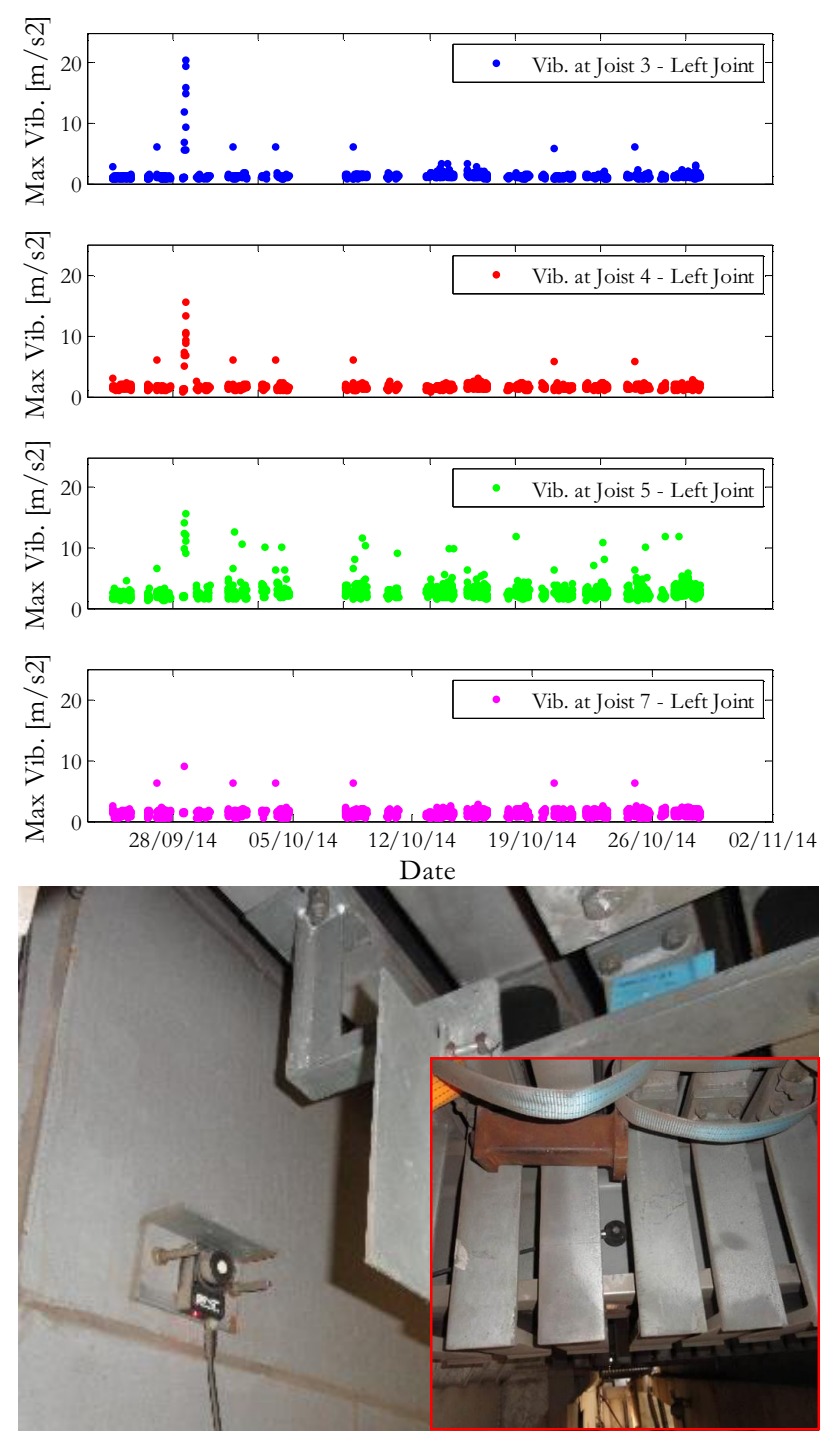

Figure 4. Example of one month's data sent to the server from the "smart" expansion joint, at four positions (top). The appearance of dense clouds of measurements outside the normal trend would indicate probable failure of a component. Accurate accelerometers fixed at a supporting joist beam and ultrasonic displacement sensors checking movements (bottom).

\section{Controlling the vibrations and cracks of a concrete box girder viaduct}

After some years in operation, the railway viaduct shown in Figure 5 had revealed cracks on the upper plate of its box girder. Every span of the concrete box girder has a total length of 35 metres, and supports two train tracks on top.

It was decided to install a monitoring system in order to observe the problem as it progressed and evaluate the influence of the overpassing rail traffic, monitoring crack behaviour under traffic and environmental loading over a period of two years. The system measures various parameters on the structure at frequencies of $100 \mathrm{~Hz}$ and $200 \mathrm{~Hz}$, using different measuring principles, and presents the data online. The system is designed to fulfil four major tasks, including monitoring of environmental 
influences (temperature, humidity), monitoring of strain behaviour, monitoring of the structure's temperature and monitoring of the vibrations of the cantilever section of the girder.

Figure 6 shows the sensor layout used to monitor the different structural parameters. A dense grid of sensors (Figures 7 and 8) has been installed in order to accurately analyse the bridge's condition.

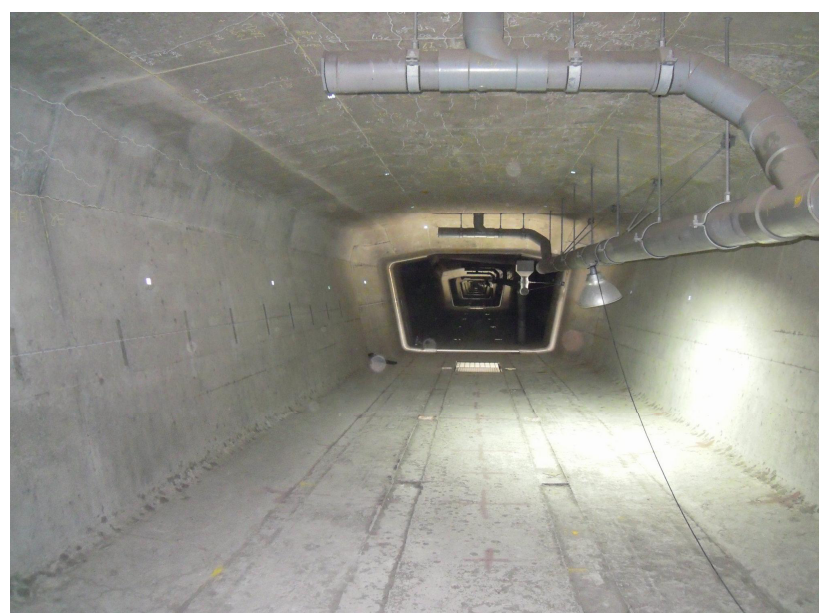

Figure 5. View of the continuous concrete box girder viaduct.

Figure 9 presents the measurements from the entire twoyear period, including environmental temperature and humidity at several locations on the bridge, structural temperature inside the concrete, micro-displacements at several locations (few are shown for clarity) and accelerations in different parts of the bridge. In general, the opening/closing movements of cracks are in line with the environmental influences. It can be observed from Figure 9 that accelerations on the deck are also in line with temperature, except for one which decreases notably. During the two-year period, there is no sign of deterioration, with no significant opening of the cracks.

It is also interesting to observe the opening of cracks due to actual loads on the bridge. Figure 10 shows this during the passage of a high-speed train. As expected, there is an evident influence on the displacement sensor but the scale of movements makes them quite negligible.

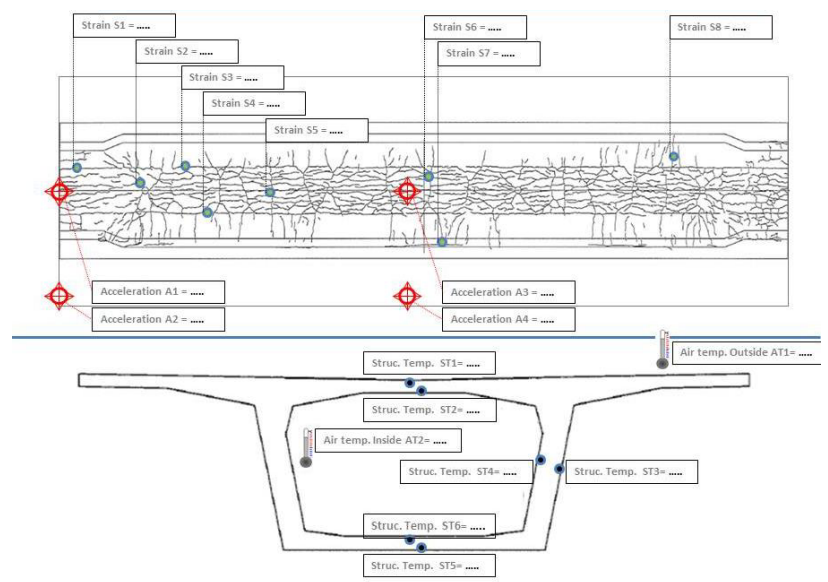

Figure 6. Layout of acceleration, strain and temperature sensors in one of the spans.

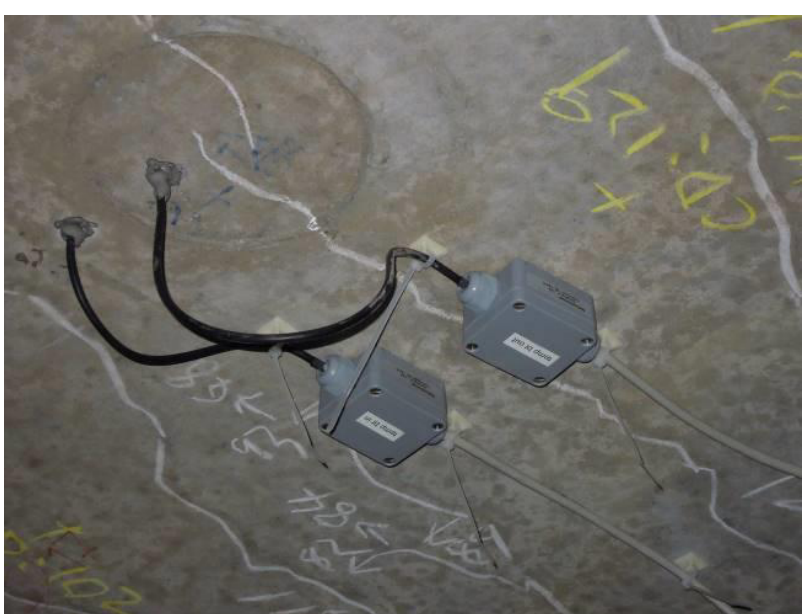

Figure 7. Structural temperature sensors installed inside the concrete structure.

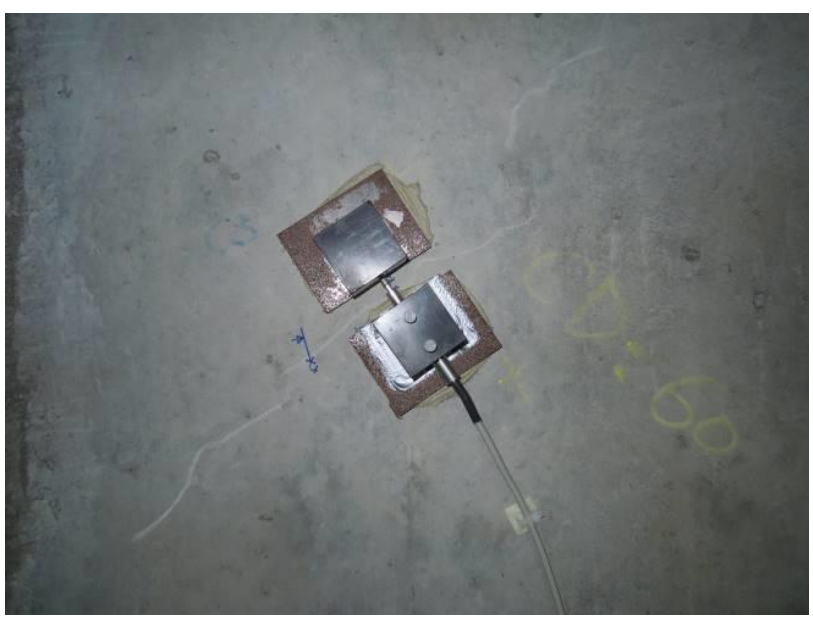

Figure 8. Crack displacement sensor installed on the upper deck of the concrete box girder.

Further data analysis has also been performed. Figure 11 shows the Root Mean Square (RMS) of continuous highfrequency $(100 \mathrm{~Hz})$ acceleration measurements during a period of five days. This is a statistical measure of the magnitude of a varying quantity, especially useful when variants are positive and negative (Islami \& Modena, 2013). The plot shows how the intensity of accelerations varies not only in accordance with the day/night intensity of trains over the bridge but also in accordance with the day/night temperature change. During the daytime, trains travel more frequently and the RMS has a high value (peak at around $10 \mathrm{am}$ ), while at nighttime, the frequency is quite low. On the other hand, the vibrations are higher when the bridge is elongating due to temperature, and lower during the night. This method of data analysis can also be applied to the actual traffic on the viaduct using accelerometers.

Figure 11 also shows the opening and closing of a crack during several days of continuous measurements. This phenomenon generally depends on temperature, but numerous maximum displacement values are also found to appear during the passage of a train. 


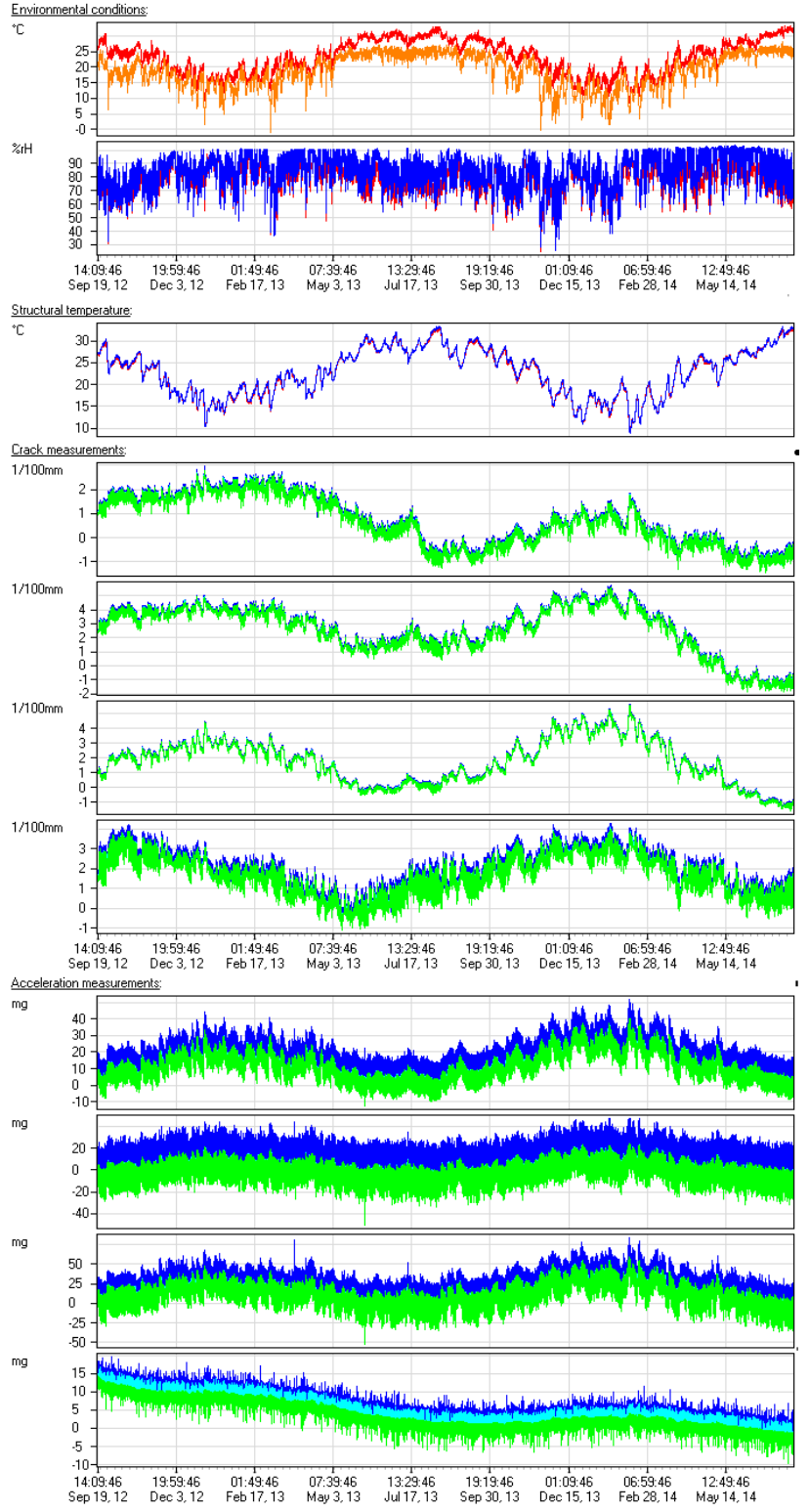

Figure 9. Measurements from two years of monitoring.
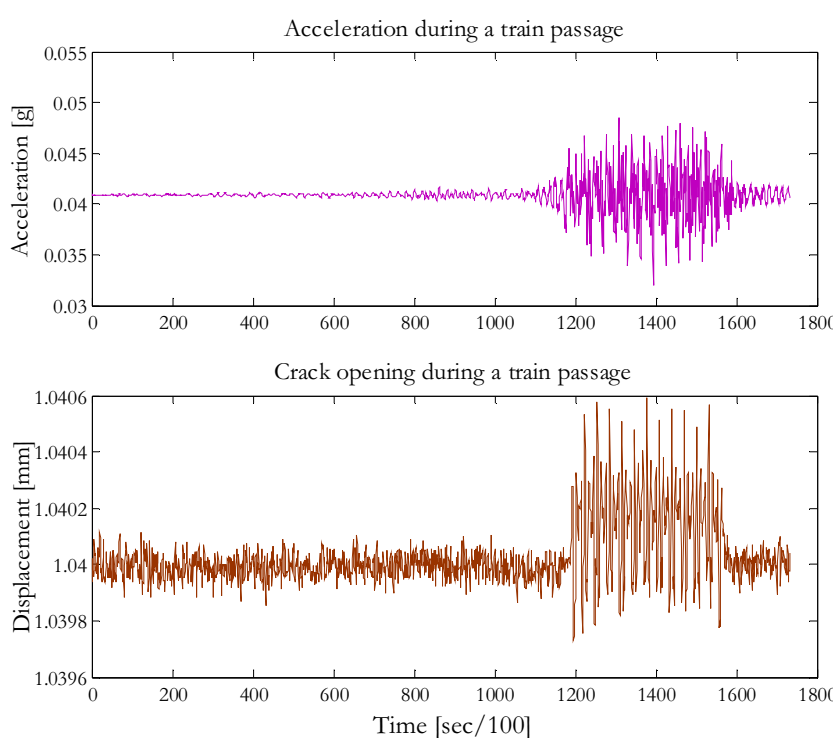

Figure 10. Train passage effects, in close view, on the accelerations and on the crack openings of the bridge.
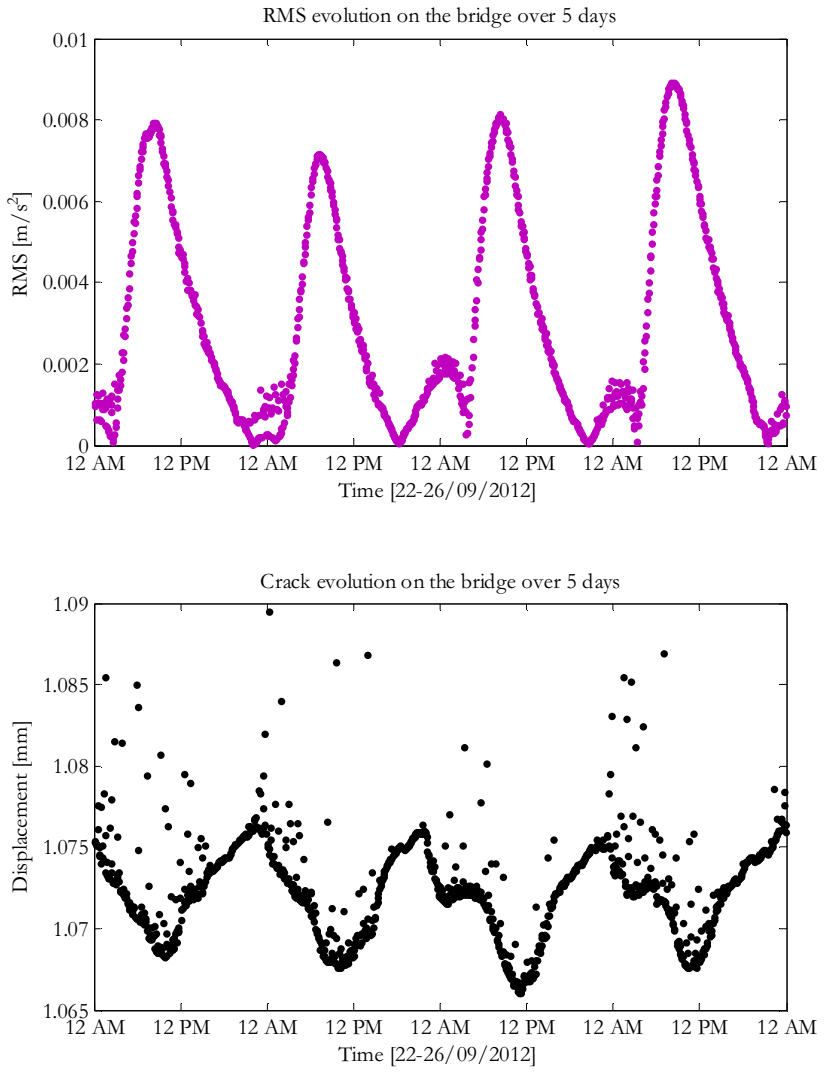

Figure 11. Continuous $100 \mathrm{~Hz}$ measurements during four days: RMS and crack opening.

The main purpose of the system was achieved by providing the responsible structural engineers with valuable data relating to vibrations and crack opening for a period of two years. The correlation of such parameters with train types and temperature could be used to determine the current state of the structure and to plan future structural refurbishment works.

System identification [2] methods have also been applied and an example is shown in Figure 12.

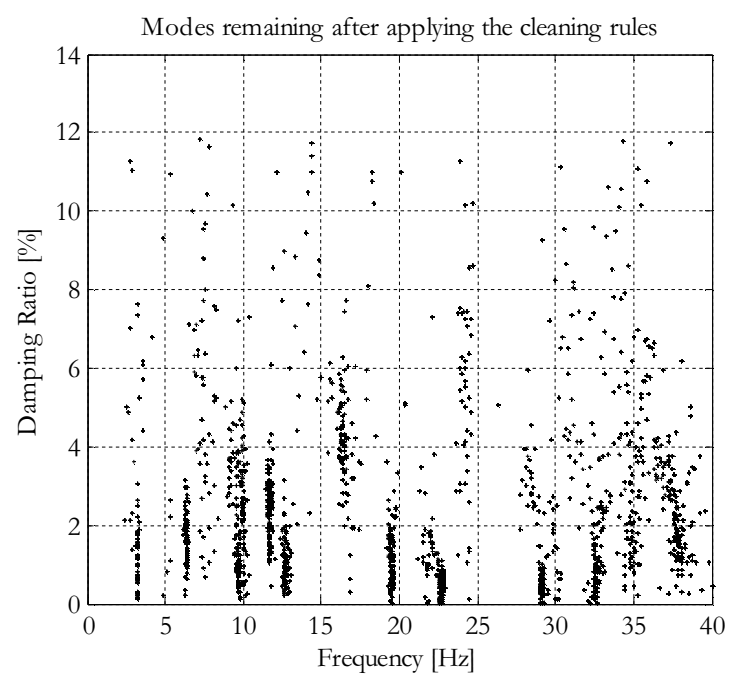

Figure 12. Stochastic system identification on the concrete viaduct: identification of $3.22 \mathrm{~Hz}, 6.35 \mathrm{~Hz}, 9.72 \mathrm{~Hz}, 11.77 \mathrm{~Hz}$ and $12.73 \mathrm{~Hz}$, etc. modes. 


\section{Dynamic monitoring of the seismic devices of a retrofitted arch bridge}

Following the recent construction of the arch bridge shown in Figure 13, which crosses a deep valley, it was decided to retrofit with lock-up devices to protect it against seismic events. The total length of the bridge is $118 \mathrm{~m}$ and the main arch span is $64 \mathrm{~m}$. The structure thus now features eight shock transmission units (STUs) at both abutments.
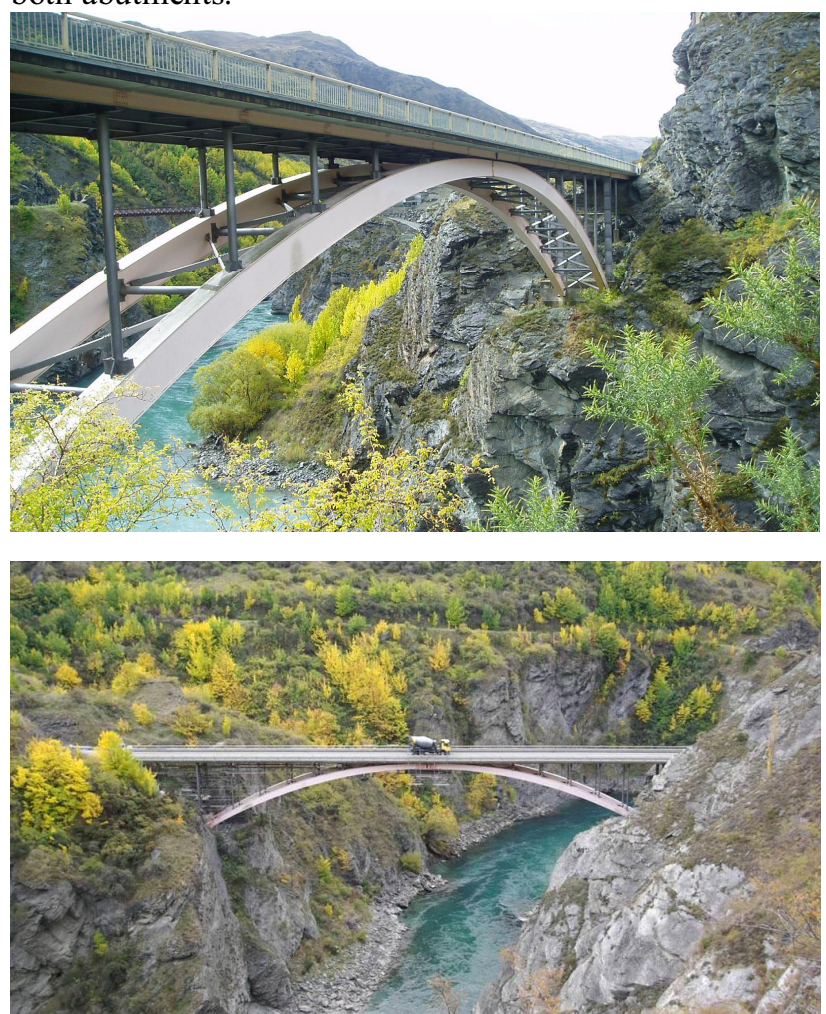

Figure 13. The monitored arch bridge, crossing a river valley.
The effect of these seismic devices is to transfer seismic loading directly to the abutments in a safe and controlled way. They do this by locking up and not allowing movements above a certain velocity, but by expanding/contracting normally with the bridge during transient everyday movement cycles. This technique is widely used by structural engineers, but now, for the first time, the performance of these devices is being monitored by a permanent automated SHM system. The system measures the pressures inside the STUs at the abutments with $100 \mathrm{~Hz}$ frequency, and correlates them with recorded seismic actions. It is also designed to issue immediate alarm notification, based on measurements by the on-structure instrumentation, should pre-defined threshold values for horizontal displacement and/or differential settlement be exceeded.

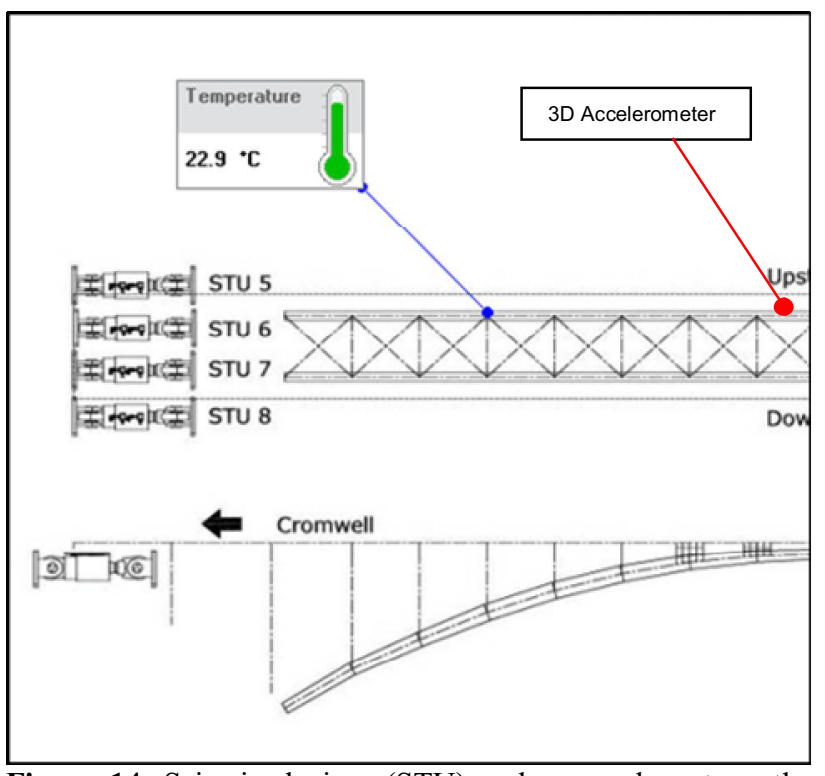

Figure 14. Seismic devices (STU) and sensor layout on the bridge.

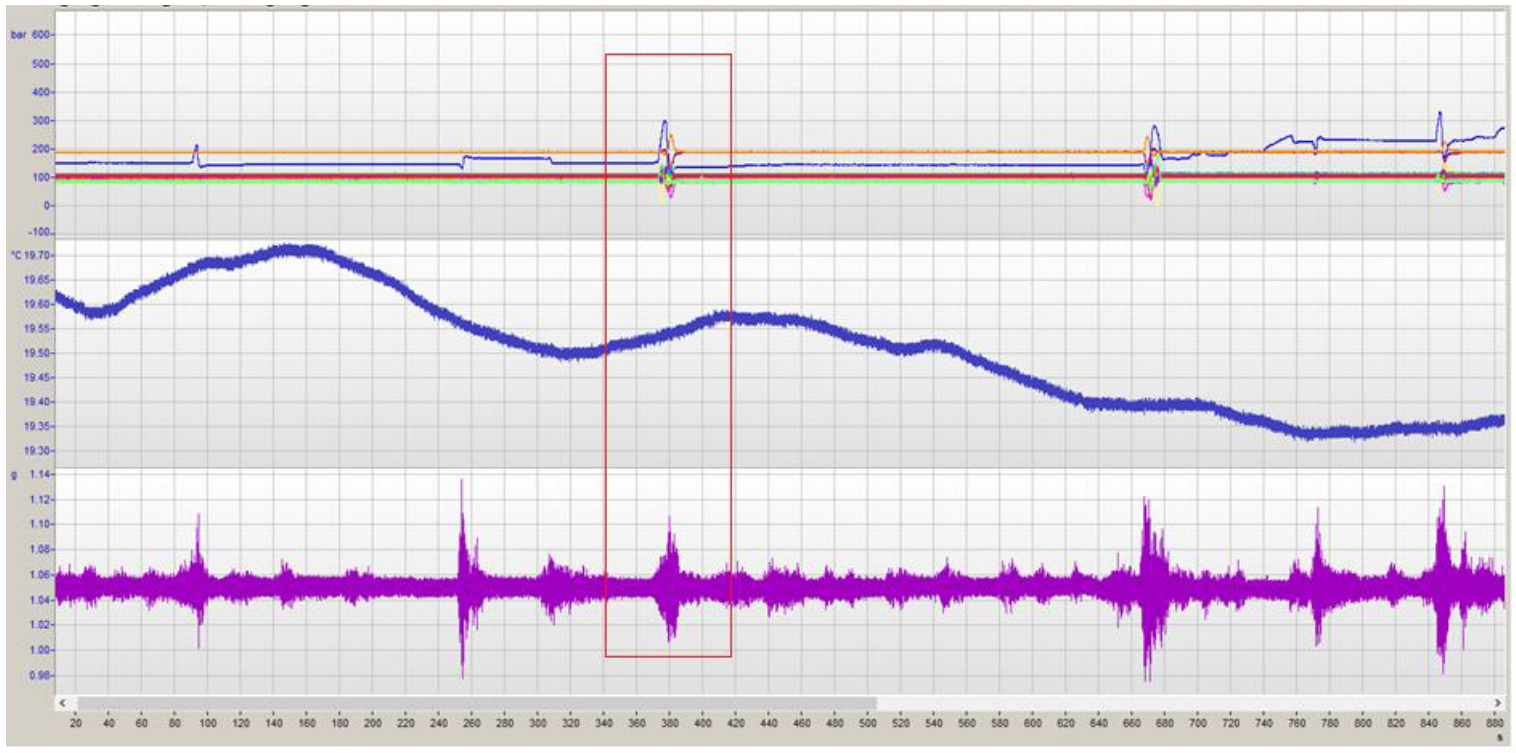

Figure 15. General plots showing $100 \mathrm{~Hz}$ data measurements and influences of traffic on seismic devices. 


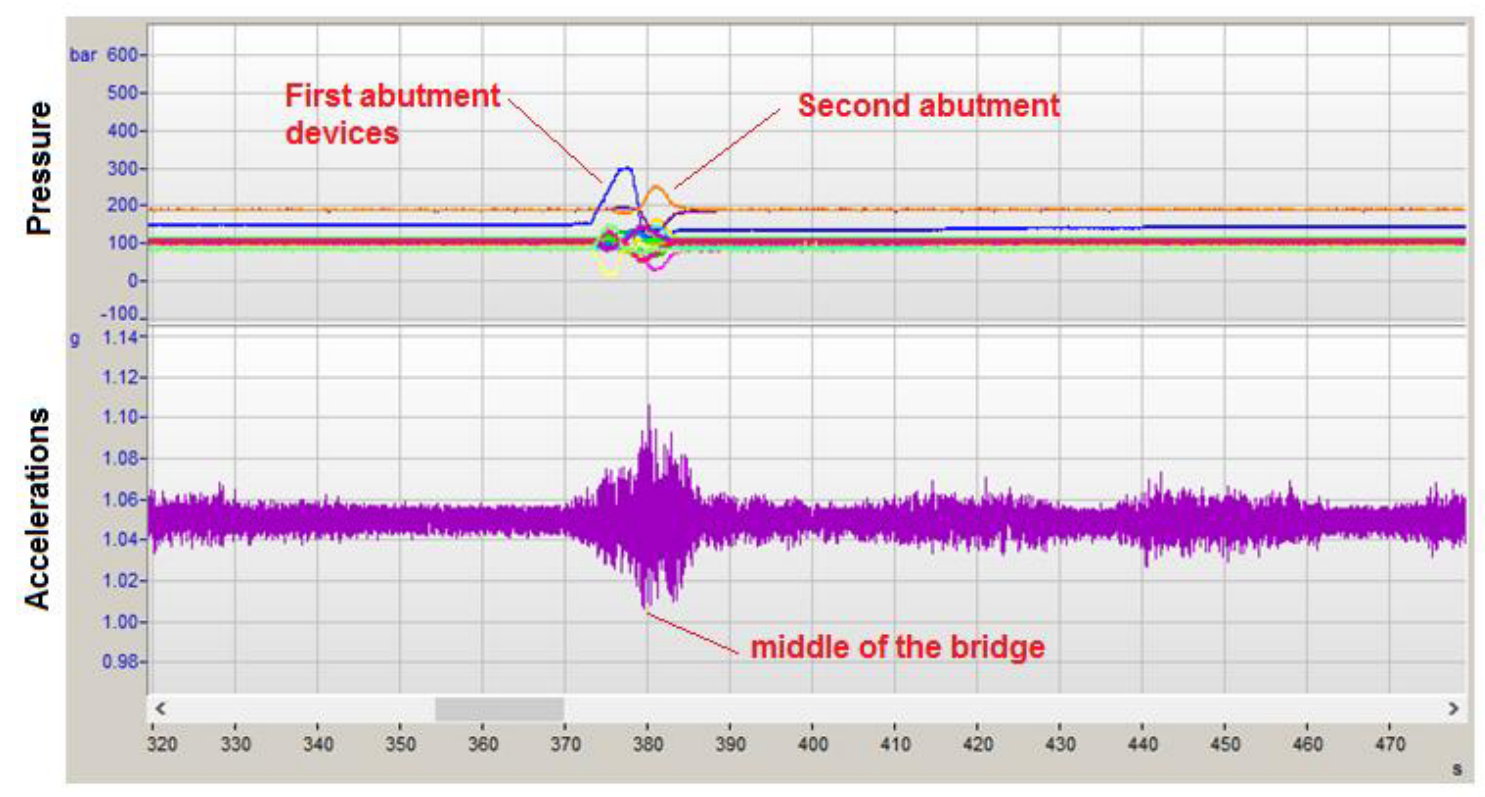

Figure 16. Close-up plots showing the correlation of pressure and acceleration during the passage of a truck.

The sensors installed on the structures include 16 pressure sensors at the STUs, plus three accelerometers and one temperature sensor which enable movements to be correlated with environmental and other influences and thus enable the data to be better evaluated.

Figure 14 shows the seismic device positions and the locations of the other reference sensors on the structure. The 16 pressure sensors inside the STUs, the 3 accelerometers and the temperature sensor are sampled at $100 \mathrm{~Hz}$ in order to detect dynamic effects of earthquakes and any other influences. A particular feature of the monitoring system is that all data is saved continuously for at least one month and, if no values have exceeded threshold values, only a limited amount of dynamic raw data is saved automatically.

Analysis of the data shows, somewhat surprisingly, that the seismic devices are sensitive also to vehicles crossing the bridge. Figures 15 and 16 show two-step zoom windows, which correlate pressure and vibrations on the structure during the passage of a heavy truck. It is interesting to notice how one can follow the route of the vehicle on the bridge by first looking at the pressures in the seismic devices of the left abutment, then the accelerations of the vibration sensor at mid-span and finally the peaks in pressure in the devices at the right abutment. This indicates that the system is working properly and that all of the seismic devices contribute to protecting the structure against external excitations.

Figure 17 shows the monitoring scheme and data transfer, and Figure 18 shows the online live presentation of all measured data during the first months of monitoring. Each graph shows the pressure values inside the chambers of the seismic devices, compared with temperature and structure vibrations.

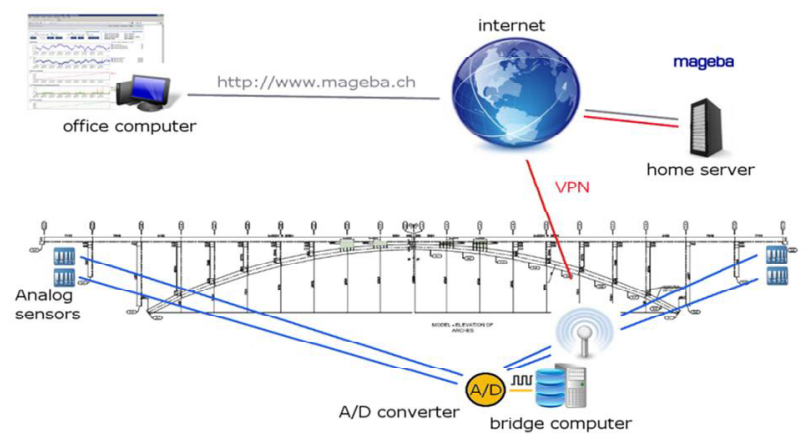

Figure 17. Monitoring layout and online data check system.

In order to get further information from the structure, other dynamic parameters such as natural frequency, damping ratio and mode shapes can also be assessed. The 3D-accelerometer at the middle of the bridge delivers optimal vibration data for the correlation of traffic or earthquake influences with pressure, and also facilitates system identification of the bridge.

Figure 19 presents the calculated frequency trend from the accelerations on the bridge. The automated analysis has detected, in particular, the first dominant mode at a frequency of approximately $4 \mathrm{~Hz}$, while other modes are slightly less dominant. During the passage of heavy traffic and earthquakes, the structural modes should be followed and any deviation might indicate damage.

The system will be in operation on site for at least two years, thus more studies will be applied to the continuous arriving data. 

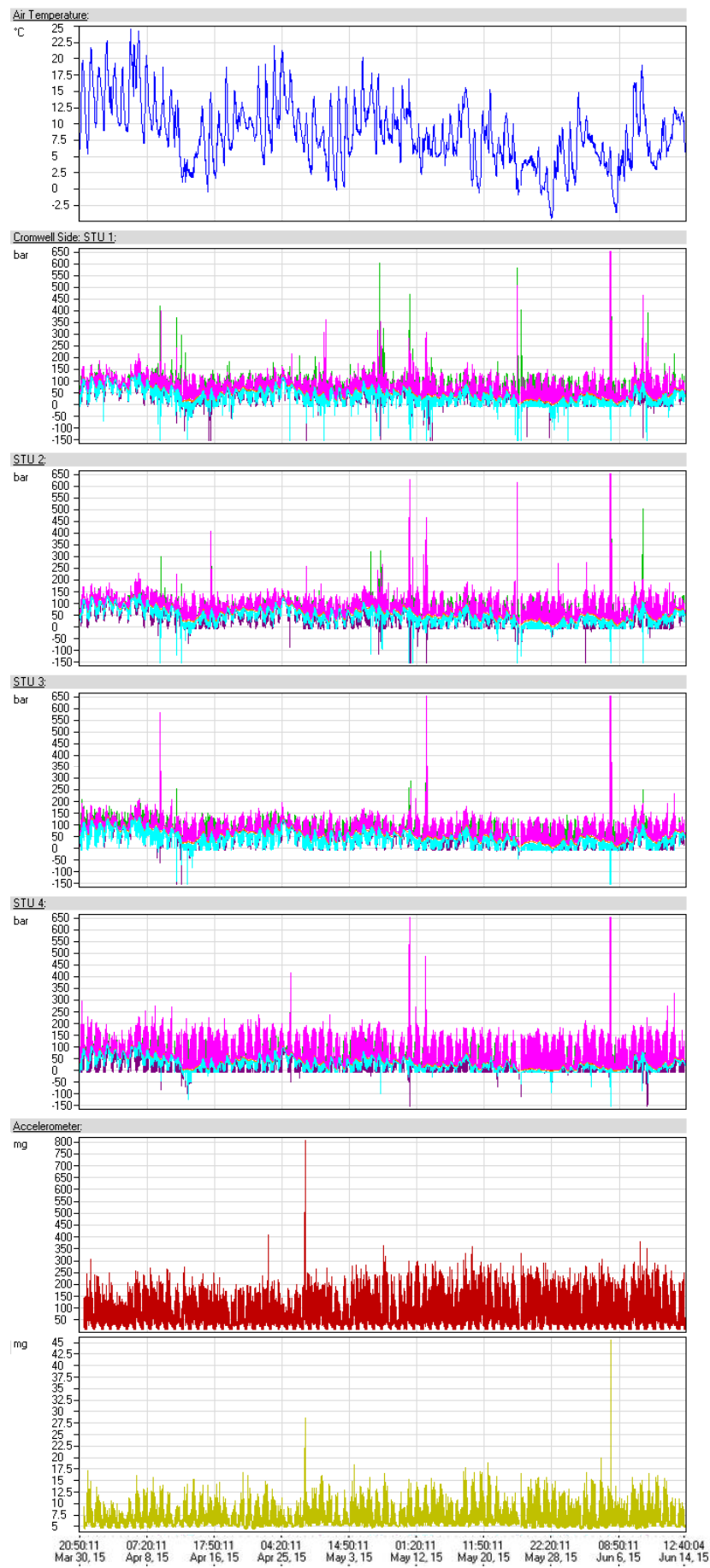

Figure 18. Plots showing all monitored data during the first months of measurements: temperature, pressure and acceleration.

$\mathrm{Hz}$

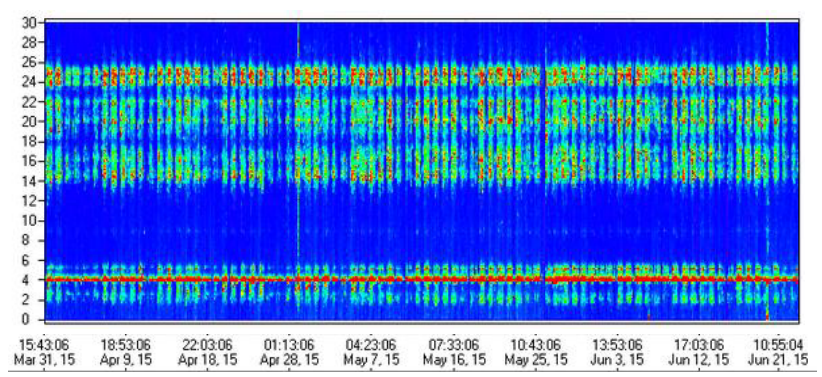

Figure 19. Time vs. Frequency graph of vertical accelerations.

\section{Conclusions}

The integration of sophisticated structural monitoring systems in newly built and existing bridges can offer great benefits to their asset management programs. Such systems can efficiently provide data required for almost any purpose, at any stage of a structure's life-cycle. They are typically much more efficient, having far lower "running costs", and are capable of an extraordinary level of detail and accuracy, e.g. in measuring high-frequency vibrations. They can also be set up to operate 24 hours a day, 7 days a week, for as long as required, and can thus be relied on to immediately record and report unexpected / serious events, no matter when they might occur.

In the presented case studies, vibration SHM systems helped to assess damage on sophisticated bridge parts, cracking and correlation with vibration on concrete structures and finally correlate vibrations with the seismic strengthening components of an arch bridge.

Whether used during installation, inspection, maintenance or replacement works, or to facilitate assessment of unexpected events or planned modifications, automated monitoring systems are thus sure to be increasingly used on bridges in years to come.

\section{References}

1. G. Moor. \& K. Islami, Monitoring and analysing the movements of suspension bridges - four case studies, International bridge conference, Pittsburgh - PA, USA (2014)

2. K. Islami \& C. Modena, Life-cycle assessment by dynamic diagnosis and long term monitoring of old bridges in North-eastern Italy, ISHMII 2013, Hong Kong (2013)

3. C. Chen, \& C. Jianchi, Taizhou triple. Bridge design \& engineering ( $B D \& E$ ) 2012, issue 66, London.

4. K. Islami et al., Life-cycle monitoring and maintenance of bridges: the role of remote SHM systems, Istanbul bridge conference, Istanbul (2014) 